\title{
Development of Monoclonal Antibodies Reactive to a New Grapevine Leafroll-Associated Closterovirus
}

\author{
Judit Monis, Agritope, Inc., Portland, OR 97224
}

\begin{abstract}
Monis, J. 2000. Development of monoclonal antibodies reactive to a new grapevine leafrollassociated closterovirus. Plant Dis. 84:858-862.

Monoclonal antibodies reactive to a previously uncharacterized grapevine leafroll-associated virus (GLRaV) coat protein were developed. A novel 37-kDa protein was found associated with grapevine leafroll disease in a mixed virus infection. The polypeptide was separated from the 38-kDa polypeptide associated with GLRaV-1 using a Tricine sodium dodecyl sulfate-polyacrylamide gel electrophoresis system. Concentrated p37 preparations were used to immunize mice for the production of monoclonal antibodies (MAb). Serum collected from the immunized mice showed reactivity with the $37-\mathrm{kDa}$ polypeptide but no reactivity with the 38 -kDa polypeptide associated with GLRaV-1 using the Western blot assay. The polyclonal antiserum reacted to both native and heat-denatured virus preparations. Two sets of anti-p37 MAbs were developed. One set binds $\mathrm{p} 37$ and also cross reacts with other GLRaV proteins, such as p36 of GLRaV-4 and -5 , while the second set recognizes p 37 specifically. We obtained the partial nucleotide sequence of the putative $\mathrm{p} 37$ viral protein. The predicted amino acid sequence revealed the presence of three out of the five conserved amino acids present in closterovirus capsid and diverged duplicate capsid proteins. We propose the name grapevine leafroll-associated virus- 8 for the new virus with a $37-\mathrm{kDa}$ capsid protein.
\end{abstract}

Additional keywords: diagnostics, ELISA, Western blot

Grapevine leafroll is a graft-transmissible disease caused by at least seven serologically distinct viruses $(1,3,11)$. Characterization of grapevine leafroll-associated viruses (GLRaVs) has been hindered by the poor quality of polyclonal antisera available, which often cannot discern between healthy and viral infected tissues by enzyme-linked immunosorbent assay (ELISA; 14). Monoclonal antibodies (MAbs) are useful because they are monospecific and generally provide better immunologic reagents for the detection of plant pathogens. MAbs have been developed with specificity to GLRaV-1 (Bioreba AG, Reinach, Switzerland), GLRaV-2 (7), GLRaV-3 (9,19), GLRaV-4, GLRaV-5 (7), and GLRaV-6 (6). Although some of the MAbs described above are commercially available, only those developed against GLRaV-1 and -3 have been useful for the

Corresponding author: J. Monis

E-mail: jmonis@wa-net.com

This research was supported in part by USDA SBIR grants 94-33610-0078 and 95-33610-1958 to J. Monis.

The nucleotide sequence reported in this paper has been deposited with GenBank as accession number AF233936.

Accepted for publication 20 April 2000.

Publication no. D-2000-0601-01R

(C) 2000 The American Phytopathological Society detection of grapevine viruses using ELISA or Western blots (14; J. Monis, unpublished data)

The LR 102 viral source is infected with a number of grapevine-associated closteroviruses (14). In this article, we describe the resolution and characterization of a newly discovered capsid protein associated with the grapevine leafroll complex disease. MAbs with reactivity to the newly discovered polypeptide (p37) associated with leafroll disease, as well as MAbs with broad spectrum reactivity to the GLRaV-4 and GLRaV-5 capsid proteins, are reported. We propose the name GLRaV-8 for the new uncharacterized virus associated with the LR 102 virus source.

\section{MATERIAL AND METHODS}

Virus sources and immunological reagents. Virus sources, except for LR 100, a grapevine cultivar infected with GLRaV-5, and Chasselas, a Swiss grapevine cultivar infected with GLRaV-2 and - $6(6,7)$, were previously described (14). The serological reagents, except for GLRaV-5 polyclonal antiserum (PAb; Sanofi Diagnostics Pasteur, Marnes-La-Coquette, France) and GLRaV-7 PAb (3), were reported elsewhere (14).

Virus extraction and purification of the 37-kDa polypeptide. Virus was extracted from different virus sources as described (14). The 37-kDa protein was isolated from the LR 102 virus preparations (14) and separated by electrophoresis using an $8 \%$ Tricine-sodium dodecyl sulfatepolyacrylamide gel electrophoresis (TricineSDS-PAGE; 17). The portion of the gel containing the $37-\mathrm{kDa}$ polypeptide was excised and eluted using an Elutetrap apparatus (Schleicher and Schuell, Moorestown, NJ). The region of the gel containing the $37-\mathrm{kDa}$ polypeptide was identified by Western blot analysis of a slab gel with GLRaV-2 USA (14) antiserum to visualize the 37- and 38-kDa viral bands. The Western blot was aligned with the remaining lanes of the gel and the region corresponding to the $37-\mathrm{kDa}$ band was excised. To ensure purity of the eluted protein, a second run on a Tricine-SDS-PAGE and elution was performed. Serological reactivity of the eluted protein was verified by Western blotting using GLRaV-1 MAb (Bioreba Ag) and GLRaV-2 USA antiserum. Concentration of the eluted protein was estimated by using the BCA protein assay kit (Pierce Chemical Co., Rockford, IL).

Preparation of MAb secreting hybridomas. Three 6- to 8-week-old female BALB/C mice were immunized intraperitoneally with the purified $37-\mathrm{kDa}$ polypeptide preparation $(7 \mu \mathrm{g})$ emulsified with Freund's complete adjuvant. Subsequently, four booster inoculations were given at 3to 4-week intervals with the same quantity of antigen intraperitoneally, in Freund's incomplete adjuvant. Anti-p37 antisera titers were monitored and the mouse with the highest titer was given final intraperitoneal and intravenous boosts of p37 without adjuvants 4 and 3 days prior to being euthanized. Immune splenocytes were subsequently collected and stored frozen before being fused to myelloma cells (P3X63-Ag8.653) to make hybridoma cell lines as described by Marusich (12). Hybridoma cell supernatants were screened using ELISA and Western blot strips as described below. Selected hybridoma cells were cloned twice, sequentially, by the limiting dilution technique, isotyped, expanded, and used to produce pure antibodies in vitro as described by Marusich (12) and Harlow and Lane (8).

ELISA. Indirect double antibody sandwich (DAS)-ELISA was used to detect virus specific antibodies. Briefly, ELISA plates were coated overnight $\left(4^{\circ} \mathrm{C}\right)$ with polyclonal antiserum (GLRaV-2 USA PAb or GLRaV-5 PAb). Concentrated extracts from infected and healthy vines (Table 1) were diluted in $0.5 \mathrm{M}$ Tris- $\mathrm{HCl}, \mathrm{pH} 8.2$, $143 \mathrm{mM} \mathrm{NaCl}, 1 \%$ polyethylene glycol 
(molecular weight [MW] 8,000), 2\% polyvinyl pyrrolidone (MW 40,000), $0.05 \%$ Tween 20 (13), and added to each sample well and incubated at room temperature for $1 \mathrm{~h}$. Each well was then filled with the hybridoma supernatants and incubated at room temperature for $2 \mathrm{~h}$. Assay plates were washed and further processed as previously described (13). Hybridoma cell lines with the lowest reactivity to healthy extracts and highest reactivity to viral antigens were chosen for further analyses.

Western blot analyses. In all experiments, either $8 \%$ Tris-SDS-PAGE (10) or $8 \%$ Tricine-SDS-PAGE (17), as described above for the separation of the $37-\mathrm{kDa}$ polypeptide, were used. For Western blot strip assay, proteins from extracts from several virus isolates were separated by Tricine-SDS-PAGE. After transfer of proteins to nitrocellulose, the blots were cut into 2-mm strips. Strips were treated with individual hybridoma supernatants from cultures of each cell line and processed essentially as previously described for a slab Western blot (14). For rapid analyses of large numbers of MAbs, a Western blot multichannel (Miniblotter 28) apparatus (Immunetics, Cambridge, MA) was used. After incubation with the MAb supernatants, the filters were washed and incubated with peroxidase-conjugated goatantimouse immunoglobulin $\mathrm{G}(\mathrm{IgG})+\mathrm{M}$ $(\mathrm{H}+\mathrm{L})$ (Pierce). Subsequently, the peroxidase was localized with the enhanced chemiluminescence immunoblotting system (Amersham Chemicals, Arlington Heights, IL).

Polyclonal antiserum production and development of ELISA for the detection of GLRaV-5 and -8. Concentrated preparation of the LR 102 virus source was used to immunize rabbits for the production of antiserum as previously described (14). The resultant antiserum is referred as LR $102-P A b$ throughout this article. A DASELISA was developed using the LR 102$\mathrm{PAb}$ as a coating serological reagent and alkaline phosphatase labeled $15 \mathrm{~F} 1 \mathrm{MAb}$ (15F1-AP) conjugate as a detection reagent. The ELISA was carried out essentially as previously reported (13).

Development of an immunoblot for the detection of a mix of grapevine-associated closteroviruses. For the simultaneous detection of seven grapevine-associated closteroviruses, concentrated prepara- tions from various virus sources were separated in $10 \%$ Tris-SDS-PAGE and transferred to nitrocellulose as described for Western blot assay. Blots were incubated with a solution containing a mix of anti-24K-RSI, anti-38-kDa, GLRaV-4 (14) polyclonal antisera, GLRaV-3 MAbs (Bioreba AG), and 15F1 MAbs (this article). After incubation with the antibody mix, the filters were washed and incubated with a mix of phosphatase conjugated goat antirabbit and goat-antimouse IgGs (Pierce) and developed essentially as described by Monis and Bestwick (14).

RNA isolation, cDNA synthesis, cloning, sequencing, and computer-assisted nucleotide and amino acid sequence analyses. Viral RNA was isolated from LR 102 virus preparations essentially as described in the RNeasy kit protocol (Qiagen, Inc., Valencia, CA) as modified by $\mathrm{X}$. Good and J. Monis (unpublished data). cDNA libraries were generated using the LR 102 viral source (X. Good and J. Monis, unpublished data). Nucleotide sequencing was performed on both strands of cDNA using the ABI Taq Dye Deoxy Terminator Cycle Sequencing kit and the ABI 373 Automated Sequencer (Perkin-Elmer Applied Biosystems, Foster City, CA). The nucleotide sequences were analyzed using MacVector DNA analyses programs. The nucleotide and amino acid sequences of

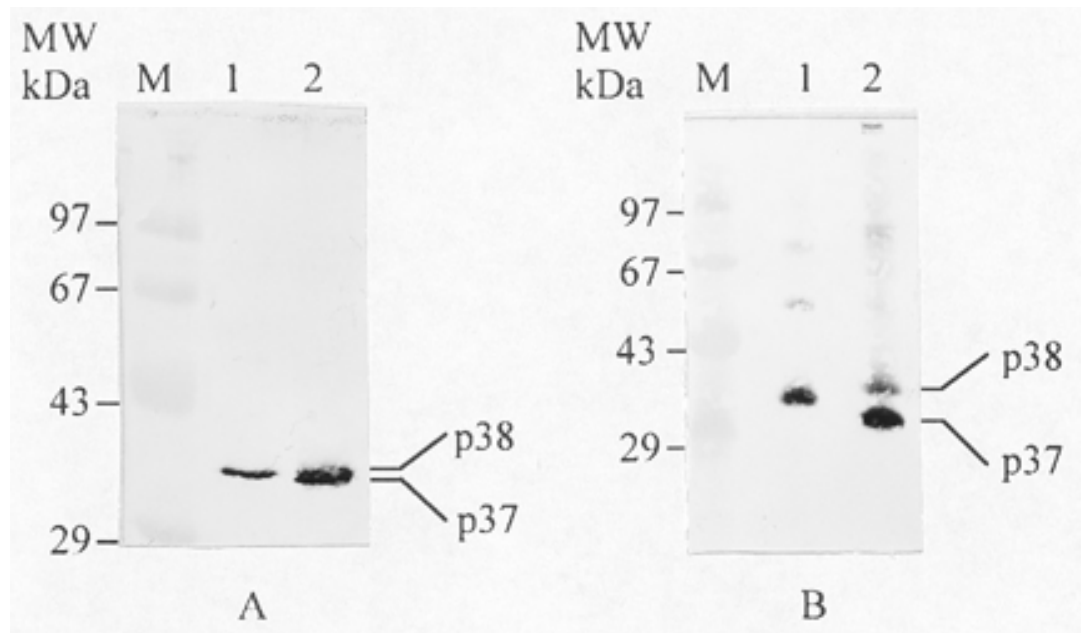

Fig. 1. Western blot analyses of (A) Laemmli and (B) Tricine-sodium dodecyl sulfate-polyacrylamide gel electrophoresis using the grapevine leafroll-associated virus-2 USA polyclonal antiserum. Lane $\mathrm{M}$, protein molecular standards; lane 1, purified virus from FC/2 (p38 virus source); and lane 2, LR 102 (p37 virus source). other closteroviruses were obtained with the Entrez program; sequence comparisons with nonredundant databases were searched for with the Blast program from the National Center for Biotechnology Information (NCBI).

\section{RESULTS}

Tricine-SDS PAGE separation of the 37-kDa polypeptide. Previous work showed that a doublet of approximately 37 to $38-\mathrm{kDa}$ polypeptides was observed when analyzing concentrated preparations of the LR 102 viral source in Western blot assays (14). In preliminary work, we used the Tris-SDS-PAGE system, which was not able to separate these proteins in spite of trying different acrylamide concentrations and gradient gels. The Tricine-SDS-PAGE allowed the resolution of two polypeptides from the LR 102 virus source doublet. The Tris-SDS-PAGE and Tricine SDS PAGE systems produced separation of $\mathrm{FC} / 2$ virus source extracts (infected with GLRaV-1) and extracts from the LR 102 virus source (Fig. 1). The blots were reacted with the GLRaV-2 USA antiserum $(2,14)$. Two polypeptides are resolved using the Tricine-SDS-system (Fig. 1B), whereas a broad band is observed with the Laemmli system (Fig. 1A). Preparative batch Tricine-SDS-PAGE was used to separate and concentrate the novel $37-\mathrm{kDa}$ poly-

Table 1. ELISA reactivity of monoclonal antibodies to grapevine leafroll-associated virus (GLRaV) sources ${ }^{\mathrm{a}}$

\begin{tabular}{lccccc}
\hline & \multicolumn{5}{c}{ Virus source } \\
\cline { 2 - 5 } Infection status & FC/2, GLRaV-1 & LR106, GLRaV-4 & LR100, GLRaV-5 & LR102, GLRaV-8 & Healthy \\
\hline AGTO cell line & & & & Neg & Neg \\
3F76 & Neg & Neg & Neg & Neg & Neg \\
5G5 & Neg & Neg & Neg & Pos++ & Neg \\
14F9 & Neg & Neg & Neg & Pos+++ \\
15F1 & Neg & Neg & Pos++ & Pos+++ \\
19A12 & Neg & Neg & Neg & Neg \\
\hline
\end{tabular}

${ }^{a}$ Samples were scored based on the $A_{405}$ readings: neg $(<0.2)$, pos+ (0.4 to 1.0), pos++ (1.0 to 2.0), and pos+++ $(>2.0)$. 
peptide for mice immunization. Gelpurified $37-\mathrm{kDa}$ polypeptide was immunoreactive with the GLRaV-2 USA PAb (Fig. 2A), but no reactivity with the GLRaV-1 MAb (Fig. 2B) was observed. In earlier work, we showed that the GLRaV-2 USA antiserum (2) had a weak reactivity to the 24-kDa polypeptide associated with GLRaV-2 (14). In the Western blots in the present work, the $24-\mathrm{kDa}$ polypeptide is almost undetectable due to the poor reactivity of this polyclonal antiserum or the lability of this viral protein. However, this protein is detectable, although at times weakly, if a polyclonal antiserum, designated as RSI-24-kDa (14), or a mix of antibodies including the RSI-24-kDa antiserum, is used for its detection as described below.

Analyses of hybridoma cell lines. Hybridoma cell lines were analyzed using ELISA and Western blot assay with antigens extracted from healthy and virus-infected grapevines as described below. Approximately 6,000 hybridoma colonies distributed in 1,920 microcultures were screened by ELISA with the LR 102 virus source antigens. Of these cultures, 16 were ELISA positive (i.e., they contained antibody reactive to the LR 102 virus source).

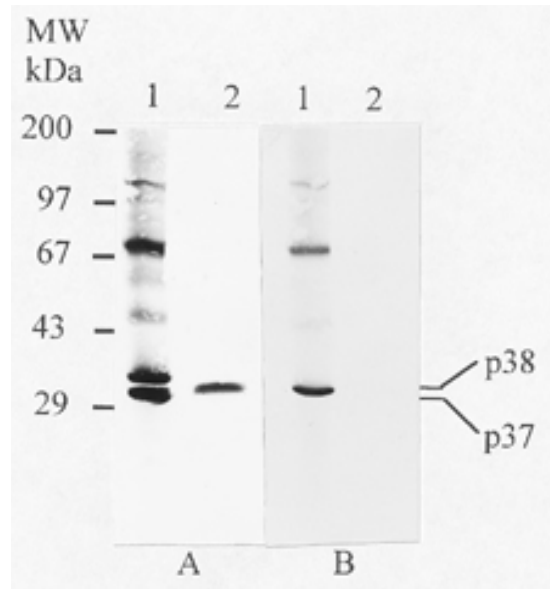

Fig. 2. Western blots of Tricine-sodium dodecyl sulfate-polyacrylamide gel electrophoresis of the LR 102 virus preparation before purification (lane 1) and with purified 37-kDa polypeptide (lane 2). (A) Grapevine leafrollassociated virus (GLRaV)-2 USA polyclonal antiserum and (B) GLRaV-1 monoclonal antibodies.
The 16 antibodies were rescreened by Western blot as described below. Further screening of ELISA-negative microcultures allowed the identification of four additional antibodies that were ELISA negative but Western blot (p37) positive. The following antigens were used in the screen: RG (a GLRaV-uninfected grapevine cultivar), LR 102 (infected with GLRaV-1, -2, -5, and -8), LR 100 (GLRaV-5), LR 106 (GLRaV-4), and Chasselas (GLRaV-2 and -6). Based on the specificity of the secondary screen, six cell lines were chosen for cloning: 3G3, 3F76, 5G5, 14F9, 15F1, and 19A12. The MAb from all cloned cell lines were determined to be from the Ig class G1, except for 5G5, which was determined to be Ig class G2a. In later screens, one of the cell lines (3G3) exhibited reactivity to all antigens tested including the uninfected control (data not shown) and was subsequently abandoned. Summaries of ELISA and Western blot analyses are shown in Tables 1 and 2 .

Monoclonal antibodies react with a novel GLRaV p37. Supernatants from the culture of the 14F9 and 19A12 cell lines were reactive in both ELISA and Western blots to the LR 102 virus source only. These MAbs did not react to antigens from GLRaV-1 (Fig. 3B), GLRaV-2 (Fig. 3C), GLRaV-3 (data not shown), GLRaV-4 (Fig. 3D), GLRaV-5 (Fig. 3E), GLRaV-6 (Fig. 3F), or healthy extracts (Fig. 3A). A virus source infected with GLRaV-7 was unavailable for our studies, but the GLRaV-7 polyclonal antiserum (3) did not react to the LR 102 antigens or any antigens from other virus sources tested.

Furthermore, the generated antibodies did not react to the LR 102 virus source after being subjected to meristem tip tissue culture to eliminate the viruses previously present (GLRaV-1, $-2,-5$, and -8).

Development of ELISA for the detection of GLRaV-5 and -8 and immunoblot for the multiple detection of grapevine-associated closteroviruses. An optimized ELISA using the LR $102 \mathrm{PAb}$ and 15F1-AP conjugates allowed the specific detection of GLRaV-5 and GLRaV-8 present in the LR 100 and LR 102 virus sources. Incubation of coating antiserum, samples, and conjugates at $4^{\circ} \mathrm{C}$ overnight were the best conditions for the detection of viral antigens. No reactivity was ob- served when crude extracts of tissues infected with GLRaV-1, -2, 3, -4, or -6 were tested.

The mixed antibody Western blot allowed the sensitive detection of GLRaV-1, $-2,-3,-4,-5,-8$, and grapevine rootstock scion incompatibility-associated virus. A typical immunoblot illustrating the reactivity of the antibody mix to various grapevine associated closteroviruses is shown in Figure 4. The faint reactivity of the antibody mix with the $24-\mathrm{kDa}$ polypeptide associated with rootstock-scion incompatibility and GLRaV-2 is due to the lability of this antigen or low concentration of this virus in infected tissues.

Sequence analyses of putative GLRaV-8 sequences. The cDNA from the LR 102 virus source generated clones with homology to GLRaV-1, -2, and -5 (J. Monis and X. Good, unpublished data). In addition, clones with no homology to any of the other known closterovirus were generated and further characterized. One cDNA clone contained a partial open reading frame that potentially codes for a 90 amino acid polypeptide. The deduced sequence suggests that this polypeptide is the putative partial closterovirus capsid or duplicate capsid polypeptide based on the presence of three out of the five amino acid signature sequences (SNR) present in other closterovirus capsid and or diverged duplicate capsid proteins $(4,18)$. The sequences of the capsid and diverged capsid proteins of GLRaV-1 (5), GLRaV-2 (18), and GLRaV-5 (X. Good and J. Monis, unpublished data) are known; therefore, we propose that the partial amino acid sequences reported here corresponds to GLRaV-8 capsid or diverged duplicate capsid protein. The partial GLRaV-8 amino acid sequence with the highlighted SNR sequence is shown in Figure 5.

\section{DISCUSSION}

In this study, five hybridoma cell lines immunoreactive with GLRaVs were cloned and characterized. Although some of the MAbs produced reacted only to denatured polypeptides, some MAbs recognized both native and denatured proteins with the same apparent specificity (e.g., the reactivity of MAbs $14 \mathrm{~F} 9,15 \mathrm{~F} 1$, and $19 \mathrm{~A} 12$ as compared in Tables 1 and 2).

Table 2. Western blot reactivity of monoclonal antibodies to grapevine leafroll associated virus (GLRaV) sources ${ }^{\mathrm{a}}$

\begin{tabular}{lcccccc}
\hline & \multicolumn{5}{c}{ Virus source } \\
\cline { 2 - 3 } Infection status & FC/2, GLRaV-1 & LR106, GLRaV-4 & LR100, GLRaV-5 & Chasselas, GLRaV-2 and -6 & LR102, GLRaV-8 & Healthy \\
\hline AGTO cell line & & & & Neg & Pos \\
3F76 & Neg & Pos & Pos & Neg & Neg \\
5G5 & Neg & Neg & Pos & Neg & Peg \\
14F9 & Neg & Neg & Neg & Neg & Pos \\
15F1 & Neg & Neg & Pos & Neg & Pos \\
19A12 & Neg & Neg & Neg & Neg \\
\hline
\end{tabular}

${ }^{\text {a }}$ Presence of virus-specific band at the expected molecular weight of the capsid protein is indicated by pos and lack of a visual viral-specific band is indicated by neg. 
Grapevine clones derived from meristem tissue culture to eliminate the viruses present in the LR 102 virus source did not display typical leafroll symptoms. The p37 was undetectable when these virus-free clones were analyzed by Western blotting using the generated MAbs. Because of the lack of serological reactivity of the 14F9 and 19A12 MAb with any other characterized GLRaVs, we propose that the uncharacterized, $\approx 37-\mathrm{kDa}$ polypeptide is the capsid protein of GLRaV-8.

The MAbs produced in this work displayed reactivities to either the $\approx 37-\mathrm{kDa}$ polypeptide alone, the $\approx 37-\mathrm{kDa}$ polypeptide and the $\approx 36-\mathrm{kDa}$ polypeptide associated with GLRaV-4 and -5 , or the $\approx 36-\mathrm{kDa}$ polypeptide associated with GLRaV-5 and the novel $\approx 37-\mathrm{kDa}$ polypeptide. Thus, the $36-\mathrm{kDa}$ polypeptide associated with GLRaV-4 and -5, and the 37-kDa polypeptide associated with the novel GLRaV-8, share at least one epitope with p37. In a previous study, we determined that, although the GLRaV-2 USA PAb (14) was able to detect a GLRaV-4 antigen, GLRaV4 PAbs were unable to detect the p37 polypeptide in Western blot (14). Interestingly, GLRaV-4 and GLRaV-5 PAbs do not crossreact with the heterologous antigens in Western blot assays or in ELISA ( $\mathrm{J}$. Monis, unpublished data).

It was not possible to determine the reactivity of the newly developed MAbs with GLRaV-7 because an isolate infected with this virus was unavailable, but the newly described $\approx 37-\mathrm{kDa}$ polypeptide associated with the LR 102 viral source was not recognized by the GLRaV-7 PAb developed by Choueiri et al. (3).

Preliminary sequence information suggests that GLRaV-8 is related to other characterized closteroviruses (X. Good and J. Monis, unpublished data). Sequence analyses of the putative GLRaV-8 viral coat protein or diverged duplicate coat protein has identified the invariant amino acid sequences $(\mathrm{S}, \mathrm{N}, \mathrm{R})$ characteristic of rod-shaped and filamentous RNA plant viruses, supporting the notion that this

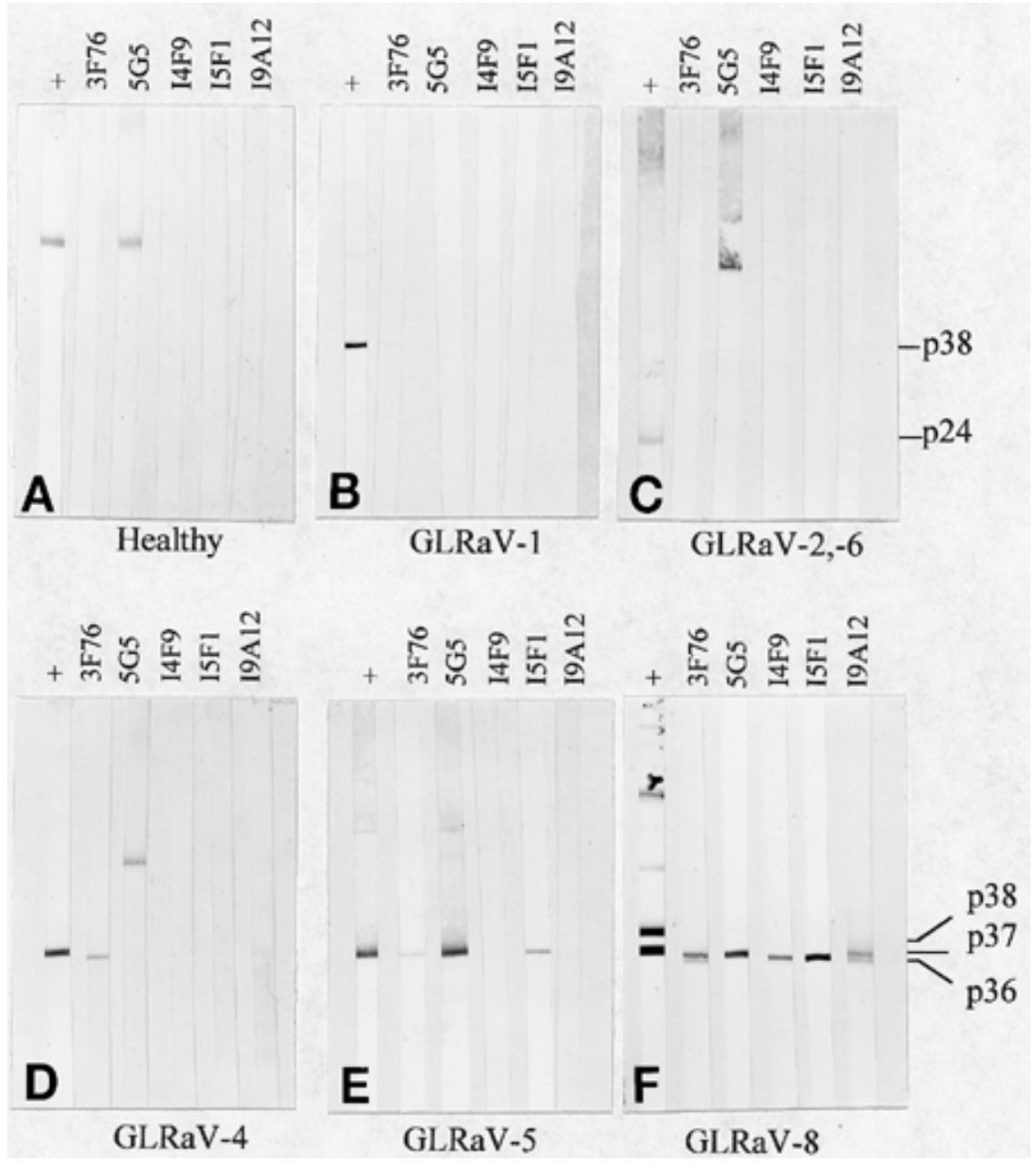

Fig. 3. Western blot analyses of monoclonal antibodies from selected cloned cell lines (3F76, 5G5, 14F9, 15F1, and 19A12). Preparative Tricine-sodium dodecyl sulfate-polyacrylamide gel electrophoresis was run with the following antigens from the following plant material: (A) Healthy; (B) grapevine leafroll-associated virus (GLRaV)-1; (C) GLRaV-2, -6; (D) GLRaV-4; (E) GLRaV-5; and (F) GLRaV-8. The (+) on top of the first strip in each group indicates a positive control reacted with the following immunological reagents (14): healthy polyclonal antiserum (PAb) (Healthy); GLRaV-1 PAb (GLRaV-1); RSI-24-kDa PAb (GLRaV-2 and -6), GLRaV-4 PAb (GLRaV-4); GLRaV-5 PAb (GLRaV-5); and GLRaV-2 USA PAb (GLRaV-8). virus belongs to the closterovirus group $(4,18)$. The sequence comparison of the putative GLRaV-8 capsid and diverged capsid protein with the homologues of GLRaV-1 (5), GLRaV-2 (18), and GLRaV5 (X. Good and J. Monis, unpublished data) indicate that these proteins share the typical SNRGD sequence found in other characterized closteroviruses (X. Good and J. Monis, unpublished data).

There are several features of the p37 protein that strongly suggest it is the capsid protein of a new member (GLRaV-8) of the closterovirus group: first, the association of $\mathrm{p} 37$ with leafroll disease; second, the large molecular weight of p37 is typical of the capsid protein of other closteroviruses that infect grapevines; third, the serological cross reactivity of GLRaV-4 and 5 capsid proteins with some MAbs generated with the p37 (shared epitopes); and, finally, the presence of conserved amino acid signature sequences found in other characterized closterovirus homologue genes. Work is in progress to clone the complete GLRaV-8 capsid and diverged duplicate capsid protein genes. The in vitro expression of the putative protein and reactivity of the recombinant protein with the MAbs reported here will allow the corroboration of the function of the putative protein gene with the corresponding sequences reported here.

The MAbs have proven useful in ELISA and Western blot assays. The developed ELISA using the 15F1 MAb and the LR $102 \mathrm{PAb}$ allowed the detection of GLRaV5 and -8 in crude plant extracts. The mixed antibody Western blot assay has been used successfully for screening grapevines for virus infection (Fig. 4). The use of the present MAbs has increased the virus specificity and sensitivity of the ELISA and Western blots by reducing the background due to the presence of host-specific antibodies in polyclonal antiserum. Although the MAbs worked poorly as trapping antibodies due to epitope specificity, viral detection was improved in ELISA

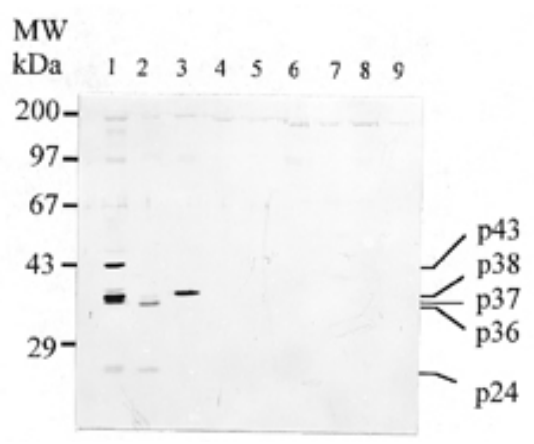

Fig. 4. Western blot using a mix of monoclonal antibodies and polyclonal antisera and the following virus sources (14): Lane 1, healthy grapevine source; lane 2, a mix of LR 102 and LR 109 virus source; lane 3, a mix of LR 106 and RSI virus source; lane 4, LR 100; and lanes 5 to 9 , uninfected grape test samples. 
Fig. 5. Partial amino acid sequence of the putative grapevine leafroll-associated virus- 8 capsid or diverged capsid protein. Note that the SNR amino acid sequence conserved in filamentous viruses is highlighted.

when the MAbs were used as conjugates. Likewise, the MAbs used in the mixed antibody Western blot reduced the nonspecific background as compared to the use of the GLRaV-2 USA polyclonal antiserum reported previously (15).

Routh et al. (16) reported inconsistent positive results with GLRaV-5 polyclonal antiserum in ELISA-immunocapture reverse transcriptase polymerase chain reaction assays using plant material infected with a mix of GLRaVs. The availability of specific MAbs to grapevine leafroll-associated closteroviruses and new molecular data will help further our understanding of the complex group of grapevine-associated closteroviruses.

\section{ACKNOWLEDGMENTS}

I thank B. Berger, F. Negru, X. Good, and A. Lammers for excellent technical support; M. Marusich and members of the Monoclonal Antibody Facility at the University of Oregon for the production and cloning of hybridoma cell lines; $\mathrm{C}$. Pico for help on figure preparation; G. P. Martelli for providing the GLRaV-7 PAb; P. Gugerli for providing the Chasselas grape cultivar infected with GLRaV-2 and -6; and G. P. Martelli, M. Marusich, and K. Eastwell for critical review of the manuscript.

\section{LITERATURE CITED}

1. Boscia, D., Greif, C., Gugerli, P., Martelli, G. P., Walter, B., and Gonsalves, D. 1995. Nomenclature of grapevine leafroll-associated putative closteroviruses. Vitis 34:171-175.

2. Boscia, D., Hu, J. S., Golino, D. A., and Gonsalves, D. 1990. Characterization of grape leafroll associated closteroviruses (GLRaV) serotype II and comparison with GLRaV III. (Abstr.) Phytopathology 80:117.

3. Choueiri, E., Boscia, D., Digiaro, M., Castellano, M. A., and Martelli, G. P. 1996. Some properties of a hitherto undescribed filamentous virus of the grapevine. Vitis 35:91-93.

4. Dolja, V. V., Boykio, V. P., Agranovsky, A .A., and Koonin, E. V. 1991. Phylogeny of capsid proteins of rod-shaped and filamentous RNA plant viruses: two families with distinct patterns of sequence and probable structure conservation. Virology 184:79-86.

5. Fazeli, C. F., and Rezaian, M. A. Nucleotide sequence and organization of ten open reading frames of the grapevine leafroll-associated virus-1 genome and identification of three subgenomic RNAs. J. Gen. Virol. In press.

6. Gugerli, P., Brugger, J. J., and Ramel, M. E. 1997. Immuno-chemical and biological distinction of grapevine leafroll associated virus 2 and 6 in complex infections with other known and unidentified viruses. Extended Abstr. 12th Meet. Int. Counc. Study Viruses Virus Dis. Grapevine, Lisbon, Portugal.

7. Gugerli, P., and Ramel, M. E. 1993. Grapevine leafroll associated virus II analyzed by monoclonal antibodies. Extended Abstr. 11th Meet. Int. Counc. Study Viruses Virus Dis. Grapevine, Montreux, Switzerland Federal Agric. Res. Stn. Changins, CH-1260 Nyon, Switzerland.

8. Harlow, E., and Lane, D. 1988. Antibodies, A Laboratory Manual. Cold Spring Harbor Laboratory Publications, Cold Spring Harbor, NY.

9. Hu, J. S., Gonsalves, D., Boscia, D., and Namba, S. 1990. Use of monoclonal antibodies to characterize grapevine leafroll associated closteroviruses. Phytopathology 80:920-925.

10. Laemmli, U. K. 1970. Cleavage of structural proteins during the assembly of the head of bacteriophage T4. Nature 227:680-685.
11. Martelli, G. P. 1997. Grapevine virology highlights. Extended Abstr. 12th Meet. Int Counc. Study Viruses Virus Dis. Grapevine, Lisbon, Portugal.

12. Marusich, M. F. 1988. Efficient hybridoma production using previously frozen splenocytes. J. Immunol. Methods 114:155-159.

13. Monis, J., and Bestwick, R. K. 1996. Detection and localization of grapevine leafroll associated closteroviruses in greenhouse and tissue culture grown plants. Am. J. Enol. Vitic. 47:199-205.

14. Monis, J., and Bestwick, R. K. 1997. Serological detection of grapevine leafroll associated closteroviruses in infected grapevines. Plant Dis. 81:802-808.

15. Monis, J., Bestwick, R. K., and Stamp, J. A. 1995. Detection of grapevine-associatedclosteroviruses by a sensitive western blot immunoassay. Am. J. Enol. Vitic. Vol. 46:404.

16. Routh, G., Zhang, Y.-P., Saldarelli, P., and Rowhani, A. 1998. Use of degenerate primers and partial sequencing and RT-PCR-based assays of grapevine leafroll-associated viruses 4 and 5. Phytopathology 88:1238-1243.

17. Schagger, H., and Von Jagow, G. 1987. Tricine-sodium dodecyl sulfate-polyacrylamide gel electrophoresis from the separation of proteins in the range of 1 to $100 \mathrm{kDa}$. Anal. Biochem. 166:368-379.

18. Zhu, H.-H, Ling, K.-S, Goszczynski, D. E., McFerson, J. R., and Gonsalves, D. Nucleotide sequence and genome organization of grapevine leafroll associated -2 are similar to beets yellows virus, the closterovirus type member. J. Gen. Virol. 79:1289-1298.

19. Zimmerman, D., Sommermeyer, G., Walter, B., and Van Regenmortel, M. H. V. 1990. Production and characterization of monoclonal antibodies specific to closterovirus-like particles associated with grapevine leafroll disease. J. Phytopathol. 130:277-288. 